\title{
Variations
}

Variations

Revue internationale de théorie critique

$20 \mid 2017$

Expériences oppositionnelles

\section{Des dockers aux damnés de la mer : néo- corporatisme et syndicalisme transnational}

\section{Arnaud Le Marchand}

\section{(2) OpenEdition}

1 Journals

Édition électronique

URL : http://journals.openedition.org/variations/794

DOI : 10.4000/variations.794

ISSN : 1968-3960

Éditeur

Les amis de Variations

Référence électronique

Arnaud Le Marchand, «Des dockers aux damnés de la mer : néo-corporatisme et syndicalisme transnational », Variations [En ligne], 20 | 2017, mis en ligne le 25 avril 2017, consulté le 25 février 2021. URL : http://journals.openedition.org/variations/794 ; DOI : https://doi.org/10.4000/variations.794

Ce document a été généré automatiquement le 25 février 2021.

Les ami•e•s de Variations 


\title{
Des dockers aux damnés de la mer : néo-corporatisme et syndicalisme transnational
}

\author{
Arnaud Le Marchand
}

1 Les dockers sont (re)devenus visibles, à la faveur de leur implication dans différents conflits, nationaux, voire globaux. On pourrait dater ce retour des grèves de Seattle (1999), lors du fameux sommet de la mondialisation néo-libérale, et plus récemment de l'implication des dockers français dans la lutte contre la Loi travail en 2016. Ces prises de position ne sont pas en soi une absolue nouveauté, mais elles font suite à plusieurs décennies d'un repli corporatiste et localiste, confinant parfois au sécessionnisme ouvrier', il est vrai à l'intensité variable d'une place à l'autre. Pourtant, dès 2001, les dockers européens vont réussir à organiser une action transnationale, coordonnée avec celle des marins, contre la directive européenne portuaire ${ }^{2}$. Cet épisode est resté connu sous l'appellation de "Guerre sur les Ports », ou "War on the Europe's Waterfront ${ }^{3}$, » selon les mots de l'International Transport Fédération (ITF). Il constitue une première réponse, toujours néo-corporatiste mais dépassant le localisme, aux stratégies des firmes et aux politiques de libéralisation du secteur. Il fut couronné par un succès syndical, mais resta relativement inaperçu par l'opinion publique. Les quelques réussites du syndicalisme docker, dans sa défense d'une organisation sociale des quais et des terminaux, si ce n'est dans celle de l'emploi, ne se sont pas arrêtées là. Il peut donc être utile de revenir sur cette histoire récente, qui semble à rebours du mouvement de déconstruction de la classe ouvrière, et de tenter une analyse de ce qui se passe dans l'ensemble de la filière du transport maritime. Cette analyse peut aider à comprendre les ressorts de cette implication des syndicats portuaires dans une lutte dépassant les frontières du secteur. 


\section{Du néo-corporatisme local au syndicalisme transnational}

2 Le traité CETA, qui entend construire un environnement favorable aux échanges entre le Canada et l'Union Européenne, prévoit certaines exceptions. L'une d'elles a reçu peu d'attention des médias ; il s'agit de la disposition dans l'article 14-1 qui concerne la manutention portuaire. Elle stipule que celle-ci n'est pas couverte par le traité quand elle est effectuée par des dockers, organisée indépendamment de l'opérateur de terminal. Si l'article définit le maritime cargo handling (la manutention portuaire) comme étant ouvert à la concurrence, son champ d'application « does not include work performed by dock labour, when this workforce is organised independently of stevedoring or terminal operator companies ». Cette référence à un « dock labour » indépendant, qui peut inclure les coopératives de dockers comme celles de Brest ou en Italie, est une première trace d'un repli face à la puissance syndicale. Cette formulation, assez vague est la reconnaissance de certaines normes spécifiques en vigueur sur les ports. Des normes qui restent soustraites au processus de convergence recherché par ce traité. La mobilisation et l'internationalisme syndical parviennent donc à retirer certains segments d'activité de la concurrence. Dans les annexes du traité se trouve aussi cette restriction, pour la Belgique, p. 1322 : «Cargo handling services can only be operated by accredited workers, eligible to work in port areas designated by royal decree ». La liste d'inscription des dockers est bel et bien maintenue, un dispositif dont le but est d'empêcher les employeurs de recourir à une main d'œuvre intermittente et sans statut, c'est-à-dire une main-d'œuvre précaire. Le fait que cette restriction concerne les ports belges n'est pas anodin. En effet le port d'Anvers est toujours cité en exemple de compétitivité et en constitue un modèle, souvent présenté comme inatteignable pour les autres ports européens, et notamment les ports français. Mais cette entorse au projet ordo-libéral transatlantique n'a fait que précéder celle au projet européen

3 Le journal Le Marin du 22 décembre 2016 annonce que l'Europe a adopté son nouveau règlement portuaire, le 14 décembre. "Sous réserve d'une validation de pure forme par le Conseil européen, la saga de quinze ans s'achève, marquée par l'échec de deux projets bien plus libéraux portés par la commission européenne en 2003 et 2006. Des manifestations musclées de dockers européens à Strasbourg avaient convaincu à l'époque les parlementaires de renoncer. La version définitive exclut d'ailleurs de l'accès au marché la manutention portuaire, renvoyée à un sage comité du dialogue social européen." ${ }^{4}$. C'est ce que signifie l'amendement adopté par le parlement européen, stipulant une exemption pour la manutention de marchandises, et spécifiant certaines dispositions relatives au transport de passagers, de l'application sur la libéralisation de la fourniture des services portuaires. Deux autres segments de l'activité portuaire, le pilotage et le dragage, sont également exclus de la concurrence, résultat obtenu par un lobbying des pilotes ${ }^{5}$. Mais la discussion reste ouverte sur le remorquage et le lamanage, cette dernière activité étant souvent assurée par des coopératives ouvrières. Cette séquence, qui intervient après une participation remarquée des dockers français à la lutte contre la «loi travail », sonne comme une victoire qui conclut deux décennies d'un cycle de lutte. En effet, les années 90 ont vu plusieurs tentatives d'en finir, en Europe, avec l'organisation du travail sur les quais, régie par les règles de la convention 137 de l'OIT, adoptée en 1947. Si la référence à cette convention fondatrice est maintenue, une dualisation du marché du travail s'esquisse avec la création des statuts bis, assortis de salaires moindres pour le travail 
dans les entrepôts des zones portuaires. Le point de départ du renouveau du syndicalisme sur les quais est sans doute la constitution de l'International Docker Council, en 2001, qui suit les solidarités nouées au moment du conflit à Liverpool, en 1995. Ce long conflit de Liverpool fut en effet le début de construction d'un syndicalisme Européen dont la caméra de Ken Loach a saisi parmi les premières étapes, dans son documentaire sur l'histoire de ce lock-out. L'IDC revendique 85000 affiliés de par le monde.

Ce média ne peut être affiché ici. Veuillez vous reporter à l'édition en ligne http:// journals.openedition.org/variations/794

Arrêt sur image du film de Ken Loach : Les Dockers de Liverpool (1997).

5 Ce qui aboutit à faire de l'identité docker, en Europe, nullement déstabilisée par les réformes des années $90^{6}$, une nouvelle fois celle d'un acteur subalterne, qu'un retournement dialectique dote d'une puissance en partie fantasmée, et sur qui repose l'identité des places portuaire face au travail «normal» ou aux relations professionnelles ordinaires, de l'industrie et des services terrestres. Cette conception d'une anomalie portuaire est d'ailleurs une constante. En 1986, j'avais travaillé le temps d'un été dans une entreprise de transit. Les salaires y étaient faibles, le stress permanent, et la pression visant à faire effectuer par le personnel des heures supplémentaires non payées, une habitude. Dans ces conditions, j'avais été étonné de constater le ressentiment très grand des salariés ordinaires contre les ouvriers dockers, au cours d'une grève qui bloquait la circulation portuaire, mais n'entrainait pour nous nulle perte de salaire, et, au contraire, nous permettait de rattraper du travail administratif en retard. La question du salaire revient souvent, notamment chez des enseignants qui considèrent anormal que les dockers aient des salaires aussi élevés, alors qu'ils n'ont pas de diplômes. C'est sous l'angle de l'échelle conventionnelle des rémunérations que les salaires des dockers sont trop élevés. Le niveau des salaires dockers étant comparable pour tous les ports européens, il n'a pas d'influence sur la compétitivité d'une place. Elle se joue sur d'autres problèmes, comme la fiabilité et les investissements et la place déjà acquise dans le réseau portuaire ${ }^{7}$. La relative inertie, voire irréversibilité, de la structure du réseau maritimo-portuaire, font que les fluctuations d'activité restent cantonnées dans un intervalle qui dépend de la position dans le réseau. Cette particularité procure un avantage aux dockers : ils ne peuvent pas être contournés aussi facilement que d'autres salariés. Car, dans une certaine mesure, leurs emplois ne sont pas délocalisables. Néanmoins, les premiers ports affiliés à l'IDC, ne sont pas les places les plus centrales du réseau portuaire mondial. En Europe, les ports allemands, néerlandais et polonais (ces derniers bénéficiant d'un glissement vers l'Est du continent de la production industrielle) ne sont pas membres, mais sont en contact avec l'IDC.

6 C'est bien l'inversion de l'ordre normal, qui est vue comme illégitime, plutôt que le résultat de ses conséquences pratiques. Au contraire, cette anormalité participe de la définition de l'identité portuaire, comme celle d'un système autonome. Ecart par rapport à la norme d'autant plus essentiel qu'il est institué, via les lois sur la manutention portuaire, après la perte du précédent marqueur de l'autonomie: les marchés à terme dans les ports, de la fin du XIX siècle à la Seconde Guerre Mondiale. Les relations conflictuelles, dans ce cadre, allant parfois jusqu'à une apparente 
inversion de la hiérarchie entre salariés et employeurs, sont de ce point de vue une fonction au sens de Norbert Elias ${ }^{8}$, pour l'ensemble portuaire, dans une période et une configuration historique donnée. C'est la reconnaissance de cette autonomie comme trait commun qui amena les employeurs et les armements à essayer d'obtenir une directive européenne, pour tous les ports et qui en retour accoucha d'une action transnationale. La mise en place d'une euro-grève et d'une euro-manifestation montre aussi que l'habitude de coopérer au niveau local, sur une base étroite, peut bien effectivement être le prélude à l'apprentissage de la coopération à une autre échelle, même si elle reste encore sectorielle. On peut aussi noter qu'elle accompagne le mouvement d'intégration des ports européens entre eux ${ }^{9}$, et l'apparition de groupes transnationaux dans la gestion des terminaux à conteneurs ${ }^{10}$. Ce qui implique que des dockers de ports différents, et de nationalités différentes, peuvent avoir le même employeur.

7 Pour autant, en 1994, quand le conflit sur la réforme de la manutention en France s'est terminé, par une relative défaite, on assistait à une nouvelle avancée des idées néolibérales, ou de celles prônant l'intégration à l'entreprise, comme mode de pacification des conflits du travail.

8 La question d'alors était de savoir si le dispositif portuaire pour organiser la flexibilité sans précarité, l'intermittence assortie d'une priorité d'embauche pour les dockers inscrits sur une liste, était soutenable. La réponse officielle était clairement négative, moins en raison du dispositif intrinsèque que de son décalage avec les normes qui devenaient dominantes, puisque au début des années 90 , de façon assez contradictoire avec la croissance des formes atypiques de travail, l'accent est mis sur l'intégration à l'entreprise pour résoudre les problèmes sociaux. La question de la pertinence de cette politique d'intégration sera posée dans les mêmes termes quelques années plus tard à propos du statut des intermittents du spectacle ${ }^{11}$, qui date lui aussi de 1947. Elle ne sera critiquée que par certains des acteurs de ce système de relations professionnelles (comme la CIP-IDF par exemple). En fait la progression des formes d'intermittence, plutôt que d'inciter à la généralisation de ces dispositifs, concourait à leur marginalisation. Des systèmes qui avaient été conçus comme une réponse à des anomalies par rapport au travail normal dans l'après-guerre, encadraient toujours ces mêmes anomalies une cinquantaine d'années plus $\operatorname{tard}^{12}$. Ils auraient pu être vus comme des pistes pour intégrer l'intermittence en développement, mais ils apparaissent alors comme des solutions instaurant des privilèges indus, alors que la précarité se répand. C'est moins leur efficacité intrinsèque que leur ajustement aux nouvelles normes, dérivant du tournant néo-libéral, remettant en cause la notion même de garanties qui pose problème. Ce qui pourrait aussi se formuler ainsi: transférer à des problèmes contemporains des solutions élaborées pour des secteurs considérés comme des anomalies en 1947 serait aussi une sorte de transfert temporel de l'anomalie. Cependant, force est de constater que ladite anomalie continue son chemin, et qu'elle pose un défi à l'idée d'une homogénéisation inévitable des normes d'embauche, des règles salariales et de l'intégration à l'entreprise. C'est à dire qu'elle a bien réussi à contrer la construction de nouvelles normes ordo-libérales sur les ports.

L'opposition de la CGT Ports et Docks à la « loi travail », s'enracine aussi dans ce refus d'intégration à une nouvelle norme libérale. En effet, une de ses conséquences est que le niveau privilégié de la négociation collective reste la place portuaire, puis le national. Négocier au sein de l'entreprise remettrait en cause cette construction. Les 
compositions d'équipes, les conditions de travail et de rémunération restent négociées à un niveau supérieur, parce que l'instabilité de l'activité implique des passages de dockers d'un terminal à l'autre, en fonction de la conjoncture. A travers leur implication dans cette lutte contre une nouvelle architecture des relations professionnelles, à travers le soutien affiché aux manifestations de "jeunes", et un message de soutien adressé à la société civile par le biais de " Nuit Debout ", s'esquisse la possibilité de coopérations nouvelles, cette fois au-delà du secteur portuaire. En effet, la visite, le 8 avril 2016, d'une déléguée de l'UL CGT du Havre à une réunion de protestation contre les violences policières, organisées à l'Université Paris 8 fut une surprise certaine. Elle suscita, en retour, une visite d'étudiants de Paris 8 , le 26 mai, à un cortège de manifestants au Havre. La vidéo de la première intervention fut regardée 39000 fois, et partagée 999 fois sur Facebook.

\section{Les marins : travailleurs intermittents globaux}

10 Les dockers ne sont pas les seuls ouvriers de la filière maritime à ne pas être intégrés à l'entreprise. En effet, les marins avec lesquels ils ont repoussé les directives européennes en $2001^{13}$ sont eux aussi des intermittents hors entreprises. L'un des principaux points rejetés par les syndicats de dockers était pourtant le refus de l'autoassistance dans la manutention, c'est-à-dire la permission faite aux armements d'utiliser leurs matelots comme dockers. C'était donc aussi une lutte pour l'emploi européen contre l'utilisation de marins "non communautaires ", à bas salaires, pour cette activité. Une lutte néo-corporatiste est donc devenue une lutte de solidarité avec les marins contre les pavillons de complaisance et la dégradation des conditions de travail à bord des navires.

11 En effet, l'évolution du travail maritime, à partir de la déconstruction des marchés internes nationaux, va suivre la voie de l'émergence d'une économie de bazar dans ce secteur. Economie de bazar déjà présente, de façon marginalisée, après la Seconde Guerre Mondiale, et qui est réveillée par la globalisation de l'emploi des marins. La crainte de manquer de marins et la recherche de baisse des coûts salariaux, contradictoires si l'on se réfère à la logique du pur marché, ont conduit les armements à développer un marché du travail professionnel dans lequel les marins ont des certificats de compétence transférables, globalisés. Compétences dont la fiabilité reste cependant problématique et dont les employeurs peuvent avoir du mal à s'assurer. L'une des conséquences de cette double stratégie, est la constitution d'équipages multiculturels, au minimum bi-nationaux ${ }^{14}$. Ce qui dans ce cas n'est que le retour de pratiques qui datent du début du XX siècle, avec l'enrôlement de travailleurs coloniaux dans les équipages anglais et français, avec une division du travail et un montant des salaires qui dépendent de la nationalité du marin, voire de son "ethnicité". Dans le récit, entre témoignage et fiction, que livre slimane Kader ${ }^{15}$, un banlieusard français d'origine algérienne est embauché à bord d'un paquebot , au salaire correspondant à la nationalité algérienne, moins élevé que celui correspondant à la nationalité française, au terme d'un entretien et d'une négociation d'embauche très rapides ${ }^{16}$. Pourtant, la crainte du rationnement de la demande de travail maritime bon marché (labour shortage) n'a pas cessé, ce qui conduit à plusieurs évolutions notables. L'une d'elles est la féminisation du secteur ${ }^{17}$. Les autres sont l'acceptation du salaire comme règle négociée, et non comme prix, et l'élaboration d'une régulation transnationale, pour 
diminuer le turn-over de la main-d'œuvre et restaurer l'image des compagnies maritimes. Cette nouvelle convention du travail maritime a été obtenue après la lutte contre les pavillons de complaisance, que les syndicats de dockers ont appuyé, en lien avec les luttes contre la déréglementation et la libéralisation des services portuaires ${ }^{18}$. Le retour de la négociation collective, sous l'égide de l'OIT, s'accompagne cependant de violations de ces dispositions. Aussi, le travail maritime est-il pris dans une double évolution, selon les segments de l'activité. D'un coté, il est un exemple de la non pertinence des modèles de pur marché, même à l'heure de la globalisation, comme en témoigne le mode de fixation des salaires, via une règle reliant son montant à la nationalité (et parfois à d'autres critères comme sur certains segments, celui de naviguer en couple) et les nouvelles normes mises en place ${ }^{19}$. De l'autre, sur ses zones périphériques, il glisse dans l'économie informelle ou celle du travail contraint.

Enfin, on peut s'interroger sur la construction de la pénurie ${ }^{20}$ (labour shortages) de maind'œuvre, aussi bien comme fait que comme perception, selon les armements, les régions, les pays ${ }^{21}$, les lignes et les catégories de travail. Construction qui peut aboutir à des perceptions très différentes selon les pays, servant de prétexte pour justifier la dégradation des conditions de travail, ou le recours au travail contraint. Certains acteurs officiels l'admettent pour les officiers, mais le relativisent pour les marins ${ }^{22}$. En effet, les pays, ou les compagnies, qui investissent dans la formation, n'ont pas de moyens légaux de retenir leurs salariés, ce qui a pour effet de moins inciter à subventionner les écoles de formation. On assiste donc à une concurrence pour retenir les personnels, notamment les officiers, qui peut pousser à la hausse des salaires. Dans le même mouvement, les certificats de compétences peuvent être accordés de façon complaisante (c'est le cas dans d'autres secteurs comme la soudure industrielle ${ }^{23}$ ). En réalité, on assiste aujourd'hui à l'émergence d'un autre modèle ${ }^{24}$, celui d'une intermittence globale, avec quelques garanties, dont la durabilité est à observer. Cela contrairement aux prévisions classiques de l'école des relations industrielles, au sujet de l'instabilité des marchés professionnels et leurs tendances à évoluer vers des marchés internes ${ }^{25}$.

De fait, le travail maritime a servi de laboratoire pour un retour de l'idéaltype du travailleur nomade sous la forme du travailleur détaché. Les évolutions en cours dans ce secteur sont donc susceptibles d'aider à comprendre les évolutions dans d'autres secteurs.

\section{Multiculturalisme syndical et Travail forcé : les damnés de la mer?}

14 Abordons maintenant un aspect plus sombre du travail maritime, à savoir le spectre de l'esclavagisme. Au tournant des années 2000, l'opinion de nombreux économistes ou sociologues travaillant sur les normes internationales du travail, et de l'ILO (OIT) est que l'interdiction de l'esclavage et du travail forcé, est le socle minimum pour mener une action internationale. Il y a convergence sur cet objectif ${ }^{26}$, car il serait difficile d'aller au-delà, en raison du relativisme culturel, et des différences de situation matérielles, qui compliquent l'obtention d'un accord sur des normes sociales plus ambitieuses. Cela apparait, pour le travail maritime, à travers l'examen d'un corpus de textes, tous en lignes, produits par des ONG, un comité gouvernemental officiel, et des organes de presse, et in fine par des universitaires. 
Une enquête aboutit à un premier rapport, publié en 2000 par une commission internationale rassemblant notamment syndicats et armateurs, qui porte le titre suivant: "Ships, slaves and competition ${ }^{27}$ » et qui décrit la dégradation des relations de travail sur certains segments, allant jusqu'au retour du travail forcé, voire de formes d'esclavage. Il propose une explication du phénomène, via la formation. : le manque de matelots et d'officiers formés résulterait de la déstructuration des marchés du travail nationaux, et le retour de l'intermittence comme forme principale d'emploi. Néanmoins, l'abus identifié comme le plus fréquent est le blacklisting des marins ayant dénoncé le non paiement des salaires. Il n'est fait mention que de cas limites de l'esclavage. Ce rapport sera une des étapes importantes pour l'élaboration de la nouvelle convention du travail Maritime (Maritime labour Convention), pilotée par l'OIT (ILO). Le répit sera de courte durée. En juin 2014, The Guardian reporte que l'industrie thaïlandaise de la crevette utilise une main-d'œuvre forcée, après d'ailleurs la publication d'un rapport coproduit par l'OIT ${ }^{28}$. Ces fruits de mer se retrouvent dans les approvisionnements de grands distributeurs comme Carrefour, Walmart, T, Cotsco et Morrison. Craignant pour son image et les réactions des consommateurs, dés Juin 2014, le groupe Carrefour annonce la suspension des achats auprès de ses fournisseurs thaïlandais. Dans ce cas, comme dans d'autres affaires similaires, il semble que l'appel à la responsabilité sociale des entreprises (RSE) constitue le moyen le plus rapide pour obtenir quelques avancées ${ }^{29}$. Néanmoins, la pratique n'a pas disparu. En mars 2015, The Associated Press publie un reportage sur le trafic de travailleurs, des immigrants sans papiers, souvent originaires de Birmanie, pris au piège dans l'industrie de la pêche thaïlandaise, et pas seulement pour le traitement des crevettes. Ces rapports sont le fruit d'enquêtes, utilisant souvent la méthodologie de l'étude de cas typique des sciences de gestion ${ }^{30}$, par des ONG soutenues par des grandes entreprises. Cela relève clairement de la politique d'appel à la RSE. Malgré le manque flagrant d'enquêtes universitaires sur ces questions, on peut signaler un article publié récemment dans "Marine Policy ${ }^{31}$ ». Les chercheurs proposent, sur la base d'une série d'entretiens, une explication du phénomène par la crainte de manquer de poissons et de main d'œuvre. Selon eux, les patrons de pêche sont confrontés à un double rationnement dû à la baisse des stocks, consécutive à la sur-pêche et à la pénurie d'ouvriers, qui leur ferait perdre des parts de marché en diminuant leur offre. Pour contourner cet obstacle, ils retiennent les pêcheurs sans papiers, ou instaurent des formes d'esclavage par la dette, ou encore paient des salaires diminués et très en retard de façon à conserver leurs marges d'exploitation. Dans cette perspective, ce quasi-esclavage résulte autant d'un impératif de rentabilité direct (diminuer les coûts) que de la volonté de maintenir, de force, la capacité de production en immobilisant la main-d'œuvre par des moyens illégaux. Ce qui suggère alors que le travail forcé peut-être une forme d'intégration forcée à l'entreprise, non assortie de droits sociaux. Ces deux formes ne vont pas forcément de pair: les salariés dockers et les marins sur les segments contrôlés se retrouvent dans une meilleure situation que ceux de la pêche, littéralement attachés à l'entreprise et à l'industrie. Selon The Guardian, une situation similaire peut se développer en Europe, notamment en Irlande ${ }^{32}$. Le gouvernement irlandais a réagi en diligentant la publication d'un rapport ${ }^{33}$. Si cette question est peu abordée dans la pêche française, cela ne signifie pas qu'elle y soit sans objet. Il y a déjà plus de dix ans, j'ai interrogé des marins algériens, qui partaient clandestinement à Boulogne pour embarquer à bord de chalutiers pratiquant la pêche hauturière, pour de longs mois. Ils étaient muni de certificats techniques en bonnes et dues formes, mais n'avaient pas de 
titres de séjours ni de permis de travail en France. L'un d'eux m'expliqua qu'il n' y avait pas de problèmes : il lui suffirait de donner une bouteille de whiskey pour passer les contrôles. Je n'ai plus jamais eu de leurs nouvelles.

Cette évolution ne concerne pas que l'industrie de la pêche. Durant la même période, du 20 août 2014 au 19 août 2015, les inspecteurs de l'ITF ont visité 7488 navires dont 2755 ne respectaient pas la Convention du travail Maritime, soit $36 \%$, un chiffre en hausse de $4 \%$ par rapport à l'année précédente, avec 371 bateaux supplémentaires. Le principal sujet de litige est le non-versement des salaires, ou le retard dans les paiements. Ces cas sont également en augmentation. Ils représentent 40 millions de dollars en 2013-2014 et 49 millions en 2014-2015 ${ }^{34}$. Phénomène qui conforte, de façon paradoxale, l'idée que le salaire n'est pas un prix, il n'est pas négocié. Dans un contexte difficile, certains armements pratiquent un rationnement quantitatif, en ne payant pas les salaires, ce qui n'est pas la même chose que de les baisser, car leur montant découle de conventions très larges. C'est-à-dire que dans le transport de marchandises, comme dans d'autres secteurs ${ }^{35}$, la tentation du recours au travail forcé et le passage à l'acte existent.

\section{Mouvements et bifurcations le long des chaînes logistiques}

17 Les chaînes logistiques font l'objet d'un investissement toujours croissant en vue de les planifier et de les garder prévisibles. Investissements dans l'automatisation et investissements de formes pour rationaliser leurs gestions. Pour autant ces chaînes se brisent, ou sont mises en péril, par des conflits sociaux. La grève reste un événement incalculable. Les modèles, mathématiques, inspirées de la théorie des jeux et les algorithmes ne peuvent l'intégrer. On considère que les conflits, salariaux par exemple, devraient être résolus avant de se produire, après un arbitrage coûts/avantages réalisés par les antagonistes. La rentabilité de la circulation planifiée est aussi éprouvée quand les consommateurs découvrent les conditions de travail, par les différents récits ${ }^{36}$, qui décrivent la logistique comme une chaîne d'esclaves, et refusent d'acheter les produits. C'est cette imprévisibilité irréductible que permettent de voir les mondes du travail portuaire et maritime.

Dans ces chaînes, la question de la coopération internationale est, on l'a vue, aussi un problème de multiculturalisme. La question de la compréhension interculturelle, entre systèmes de droits et d'action différents, est aussi importante pour l'International Docker Council que pour l'ITF. Les délégués syndicaux ne manquent pas d'y faire référence, comme une des difficultés, et aussi l'un des intérêts de cette construction internationale. Un permanent de la CFDT Marins et représentant de l'ITF que j'ai interrogé était particulièrement ému de l'effort de traduction de normes et de conceptions auquel il avait assisté lors de négociations avec des syndicalistes chinois, pour l'élaboration de la convention du travail maritime ${ }^{37}$. Ce qui au passage mettait, de facto, les membres de la CFDT marins en délicatesse avec la promotion de la négociation d'accords d'entreprise par la «Loi travail» française, soutenue par leur Confédération. Car c'est en portant la négociation à son échelle la plus large, que des améliorations sensibles ont été obtenues par les marins ${ }^{38}$. Loach. Les syndicalistes français anglais et portugais qui assistèrent aux «troisièmes 
journées Jules Durand » organisées par le Cirtai à l'Université du Havre en novembre $2016^{39}$, y firent plusieurs fois référence lors des débats. Le dialogue (voire le management) interculturel fait donc partie des tâches du syndicalisme dans la globalisation. Ce qui peut aussi bien déboucher sur un néo-corporatisme globalisé, toujours sectoriel, que sur une ouverture vers d'autres questions sociales et politiques. Cela peut déboucher sur une sorte de responsabilité sociale du syndicalisme néocorporatiste, prenant en charge des externalités pour protéger son image, de façon analogue à ce qui s'est produit pour les grandes entreprises multinationales, et qui finalement trouverait sa place dans le néo-libéralisme ${ }^{40}$. A moins que l'automatisation de ces activités, allant jusqu'à envisager la mise en service de bateaux-robots, qui a clairement pour but de contourner les travailleurs non délocalisables (dont les salariés du transport), ne parviennent à devenir une solution globalement rentable. Elle ferait alors disparaître ainsi toute forme syndicale. L'incertitude intrinsèque au processus de circulation liée aux mouvements sociaux compte parmi les principaux obstacles à la réussite de ce projet technocratique, encore que la question de son financement puisse aussi s'avérer délicate. Ce scenario peut aussi accompagner un dépassement politique de la situation, un au-delà du néo-corporatisme transnational et de ses marges, car il ne concerne évidemment pas seulement les dockers et les marins. Mais c'est bien la pertinence de la construction d'un syndicalisme global qui semble être le principal message adressé par les dockers aux opposants à la Loi Travail.

\section{NOTES}

1. Xavier Vigna, Histoire des ouvriers en France au XX ${ }^{\mathrm{e}}$ siècle, Perrin, 2012, $404 \mathrm{p}$.

2. Aurélie Decoene, «La libéralisation des services portuaires et la grève des dockers ", Courrier hebdomadaire du CRISP 2007/21 ( ${ }^{\circ}$ 1966-1967), p. 5-89. 2007

3. Turnbull P. The War on Europe's Waterfront - Repertoires of Power in the Port Transport Industry. British Journal of Industrial Relations [serial online]. June 2006;44(2):305-326 2006.

4. Le Marin, art. cité p 16 article Thibaud Teillard, 22 décembre 2016.

5. Néanmoins les dockers espagnols lance un mouvement de grève contre la loi portuaire espagnole le 28 février 2017, à l'appel de la coordination nationale des travailleurs de la mer $\left(\mathrm{CETN}^{\circ}\right.$.xxx, en conséquence, le 16 mars les députés espagnols ont rejeté le projet de loi du gouvernement conservateur. In le Marin 23 mars 2017, p15.

6. Pigenet Michel, «Les dockers. Retour sur le long processus de construction d'une identité collective en France, xixe-xxe siècles ", Genèses, 1/2001 ( $\left.\mathrm{n}^{\circ} 42\right)$, p. 5-25.

7. Le Marchand, A., Joly, O. Regional integration and maritime range. In Ports in Proximity: Competition and coordination among adjacent seaports. Publié par Theo Notteboom, University of Antwerp, Belgium, César Ducruet, Paris-I Sorbonne University, France and Peter de Langen, Eindhoven University of Technology, The Netherlands. Ashgate, Octobre 2009.

8. Norbert Elias, «Qu'est ce que la sociologie » Agora Pocket 1993, p 91.

9. César Ducruet, Martijn Van Der Horst. Transport integration at European ports: Measuring the role and position of intermediaries. European Journal of Transport and Infrastructure Research, Delft University of Technology, 2009, 9 (2), pp.121-142. <halshs-00458591> 
10. Michèle Collin, Thierry Baudoin, Arnaud Le Marchand. Global democracy in embryo, On the pioneering role of European harbour cities. Texte original disponible sur http:// www.eurozine.com/articles/2012-09-10-marchand-en.html. Tradu.. 2012. <hal-00798658>

11. Menger Pierre-Michel. Les intermittents du spectacle. Espaces Temps, 82-83, 2003. Continu/ Discontinu. Puissances et impuissances d'un couple. pp. 51-66;

12. A. Le Marchand, « Le syndicalisme docker au Havre depuis 1947 ; de l'action structurante à la double contrainte », Cahiers du Grhis, $n^{\circ}$ 7, 1997,

13. Aurélie Decoene, art .cité.

14. Arnaud le Marchand, "L'habitat des gens de mer », in Cousin Grégoire, Loiseau Gaëlla, Viala Laurent, Crozat Dominique, Lièvre Marion (dir.) (V1: 11 janvier 2016). Actualité de l'Habitat Temporaire. De l'habitat rêvé à l'habitat contraint, collection «SHS », Terra HN éditions, Marseille

15. Slimane Kader "Avec vue sous la mer" Allary Editions 2014.

16. Slimane Kader op.cité p 18.

17. Belinda Johnson, Mariam Seedat Khan, Joseph Rudigi, «Identity Management Strategies : Gender Based Challenges at Sea ", Journal of Gender and Religion in Africa, vol. 19, \#2, décembre 2013.

Jo Stanley Women at Sea, 1750-today: From Cabin 'Boys' to Captains, History Press, Stroud, 2016

18. Lillie N. Global Collective Bargaining on Flag of Convenience Shipping. British Journal of Industrial Relations [serial online]. March 2004;42(1):47-67. 2004.

19. Flecher C. (2013), « " Change your mind, be safe! » ", Variations [En ligne], 18 | 2013, mis en ligne le 31 mai 2013, consulté le 06 mars 2014. URL : http://variations.revues.org/636

20. | Priscilla Léong PhD Thesis; Understanding the Seafarer Global Labour Market in the Context of a Seafarer 'Shortage Cardiff University, 2012.

21. Ying Wang,Gi Tae Yeo The Selection of a Foreign Seafarer Supply Country for Korean Flag Vessels The Asian Journal of Shipping and Logistics December 2016.

22. Voir ainsi : Manpower report.The global supply and demand for seafarers. International Chamber of shipping. http://www.ics-shipping.org/docs/default-source/resources/safety-security-andoperations/manpower-report-2015-executive-summary.pdf?sfvrsn=16

23. Lefebvre Bruno, Ethnographie des travailleurs en déplacement. Voyages en Europe sociale, Paris, L'Harmattan, coll. « Logiques sociales », 2012

24. Arnaud Le Marchand, "La convention du travail maritime et le marin connecté ", Netcom, 29-1/2, 2015, 133-152.

25. David Marsden Marchés du travail : limites sociales des nouvelles théories, Paris, Économica, 1989.

26. Bhagwati, J., (1998), A Stream of Windows: Unsettling Reflections on Trade, Immigration and Democracy Cambridge: MIT Press, 1999; R. Mac Intyre 2008« Are workers rights human rights?» Michigan Universitary Press 2008

27. Consultable à ce lien http://www.itfglobal.org/seafarers/icons-site/images/ICONSfullreport.pdf

28. International Labour Organization and Asian Research Centre for Migration, Employment Practices and Working Conditions in Thailand's Fishing Sector,ILO,Bangkok, 〈http://www.ilo.org/ public/libdoc/ilo/2013/482174.pdf), 2013.

29. Locke, Richard, Fei Qin, and Alberto Brause. 2006. "Does Monitoring Improve Labor Standards? Lessons from Nike." Corporate Social Responsibility Initiative, Working Paper No. 24. Cambridge, MA: John F. Kennedy School of Government, Harvard University

30. International Labor Rights Forum (ILRF), Migrant Workers Rights Network ( MWRN) How workers can lead sustainable change inThailand's seafood processing sector Building a right culture mars 2016.

31. Supang Chantavanich a, Samarn Laodumrongchai Christina Stringer Under the shadow: Forced labour among sea fishers in Thailand Marine Policy 68. 2016. 
32. Revealed: trafficked migrant workers abused in Irish fishing industry The Guardian 2 novembre 2015.

33. Report of the governement task force on non-EEA workers in the Irish fishing fleet December 2015, http://www.agriculture.gov.ie/media/migration/publications/2015/TaskForceReport141215.pdf 34. Jérôme Hervé Droit des marins souvent bafoués Le marin 18 /09/15, p 6 et source ITF.

35. Bernardot Marc, Cousin Grégoire, Le Marchand Arnaud, Mésini Béatrice, «Camp et campements. Des économies aux principes opposés ", Multitudes, 3/2016 (n 64), p. 92-99.

36. Christian Salmon, Storytelling la machine à fabriquer des histoires et à formater les esprits, 240 p., La Découverte, 2007.

37. Entretien juin 2016.

38. Nathan Lillie A Global union for global workers: Collective Bargaining and Regulatory Politics in Maritime \$hipping , New York Routledge 2006.

39. Organisés notamment par John Barzman dont on consultera John Barzman, Dockers, métallos, ménagères. Mouvements sociaux et cultures militantes au Havre (1912-1913). Publications des universités de Rouen et du Havre, 1997 ainsi que : JULES DURAND Un crime social et judiciaire Sous la direction de John Barzman et Jean-Pierre Castelain - Université du Havre Les Amis de Jules Durand L' Harmattan 2016.

40. Colin Crouch L'étrange survie du néo-libéralisme. Diaphanes 2016.

\section{RÉSUMÉS}

La participation des dockers au conflit sur la « Loi Travail » est l'occasion de revenir sur les luttes de ces dernières décennies dans le secteur maritimo-portuaire. Elles ont accompagné la formation d'un syndicalisme transnational, coopérant avec le syndicalisme global des marins, ce qui esquisse une coopération plus générale. Cependant sur certains segments du secteur, la dégradation est telle que c'est par l'action des ONG que les luttes sociales peuvent se mener, par l'appel à la responsabilité sociale d'entreprise et au boycott par les consommateurs.

\section{INDEX}

Mots-clés : relations industrielles, économie maritime, travail forcé, syndicalisme, mondialisation.

\section{AUTEUR}

\section{ARNAUD LE MARCHAND}

Maître de conférence en science économique à l'Université du Havre, ses recherches portent sur les travailleurs mobiles, les habitats non ordinaires et l'hybridation entre économie de bazar et économie sociale dans la mondialisation. 